\title{
Relevance of Ultrastructural Alterations of Intercellular Junction Morphology in Inflamed Human Esophagus
}

\author{
Chia-Chin Liu, ${ }^{1}$ Jeng Woei Lee, ${ }^{1}$ Tso-Tsai Liu, ${ }^{2}$ Chih-Hsun Yi ${ }^{2}$ and Chien-Lin Chen ${ }^{2 *}$ \\ ${ }^{1}$ Department of Life Sciences, Tzu Chi University, Hualien, Taiwan; and ${ }^{2}$ Department of Medicine, Buddhist Tzu Chi General Hospital and Tzu \\ Chi University, Hualien, Taiwan
}

\section{Background/Aims}

Detailed characterization of the ultrastructural morphology of intercellular space in gastroesophageal reflux disease has not been fully studied. We aimed to investigate whether subtle alteration in intercellular space structure and tight junction proteins might differ among patients with gastroesophageal reflux disease.

\section{Methods}

Esophageal biopsies at $5 \mathrm{~cm}$ above the gastroesophageal junction were obtained from 6 asymptomatic controls, 10 patients with reflux symptoms but without erosions, and 18 patients with erosions. The biopsies were morphologically evaluated by transmission electron microscopy, and by using immunohistochemistry for tight junction proteins (claudin-1 and claudin-2 proteins).

\section{Results}

The expressions of tight junction proteins did not differ between asymptomatic controls and gastroesophageal reflux disease patients. In patients with gastroesophageal reflux disease, altered desmosomal junction morphology was only found in upper stratified squamous epithelium. Dilated intercellular space occurred only in upper stratified squamous epithelium and in patients with erosive esophagitis.

\section{Conclusions}

This study suggests that dilated intercellular space may not be uniformly present inside the esophageal mucosa and predominantly it is located in upper squamous epithelium. Presence of desmosomal junction alterations is associated with increased severity of gastroesophageal reflux disease. Besides dilated intercellular space, subtle changes in ultrastructural morphology of intercellular space allow better identification of inflamed esophageal mucosa relevant to acid reflux.

\section{(J Neurogastroenterol Motil 2013;19:324-331)}

\section{Key Words}

Extracellular space; Gastroesophageal reflux; Microscopy, Electron, Transmission; Tight junctions

Received: April 11, 2013 Revised: April 30, 2013 Accepted: May 3, 2013

(c) This is an Open Access article distributed under the terms of the Creative Commons Attribution Non-Commercial License (http://creativecommons. org/licenses/by-nc/3.0) which permits unrestricted non-commercial use, distribution, and reproduction in any medium, provided the original work is properly cited.

*Correspondence: Chien-Lin Chen, MD, PhD

Department of Medicine, Buddhist Tzu Chi General Hospital and Tzu Chi University, 707, Section 3, Chung-Yang Road, Hualien 970 , Taiwan

Tel: +886-3-8561825, Fax: +886-3-8577161, E-mail: harry.clchen@msa.hinet.net

Financial support: This study was supported by a grant (No. TCRD100-17) from Buddhist Tzu-Chi General Hospital, Taiwan.

Conflicts of interest: None (A part of this study was presented as a poster presentation at the DDW 2012 in San Diego and published as an abstract form in Gastroenterology 2012;142(5 suppl 1):S754).

Author contributions: Chien-Lin Chen and Chia-Chin Liu: study concept and design, analysis and interpretation of data, drafting of the manuscript, statistical analysis, and approval of the final version of the manuscript. Jeng Woei Lee, Tso-Tsai Liu and Chih-Hsun Yi: acquisition of data and research performance, and approval of the final version of the manuscript. 


\section{Introduction}

Gastroesophageal reflux disease (GERD), with typical presentation of heartburn and acid regurgitation, is a common disorder with complex definitions in the 21 th century. ${ }^{1}$ Its atypical presentation includes asthma, chronic cough, or chronic laryngitis. ${ }^{2}$ It can be further categorized based on esophagogastroduodenoscopy (EGD) into erosive esophagitis (EE) and non-erosive reflux disease (NERD), in which the prevalence of the latter group was reported to be around $50 \% .^{3}$

Overt reflux associated with esophageal injury occurs when there is a failure in the esophageal defense mechanisms, which may include limiting reflux frequency, reducing acid contact time between epithelium and acid, and tissue resistance against injury by acid. ${ }^{4}$ Of these defense mechanisms, tissue resistance, mainly at tissue level, consists of a mucus- and bicarbonate-containing unstirred water layer, the apical membrane of esophageal mucosa, the intercellular junctional complexes, bicarbonate buffering, and $\mathrm{H}^{+}$ion transporters located on the basolateral membrane. ${ }^{4}$ The intercellular junctional complexes allow cells to adhere more tightly to each other by bridging protein connections mediated by claudins and occludins for the tight junction, e-cadherin for the adherens junction, and desmocollin and desmoglein for the desmosomes. $^{5,6}$

The claudins constitute a family of 24 distinct transmembrane proteins that are composed of 4 transmembrane domains and two extracellular loops, which are involved in the homophilic and heterophilic interactions with other adjacent claudins. ${ }^{7}$ Claudins display distinct patterns of expression that are tissue-specific ${ }^{8}$ and account for much of the diversity in transepithelial permeability of the tight junction, ${ }^{9}$ and such differences also lead to varying degrees of vulnerability of the tight junction to diseases. Alterations in tight-junction proteins (TJP) may increase the permeability of the esophageal mucosa, therefore impairing tissue resistance of esophageal epithelium. ${ }^{10}$ It is yet unclear whether the expression of these proteins is altered in reflux esophagitis in humans.

The dilatation of intercellular space (ICS) has been suggested to be a pathological marker for acid related esophageal damage based on studies in rabbit esophageal mucosa ${ }^{11,12}$ and human esophageal mucosa. ${ }^{13}$ This subtle change is poorly identified on light microscopy (LM), but easily visualized by transmission electron microscopy (TEM). Subsequently, it is observed in association with bile reflux, ${ }^{14}$ NERD, ${ }^{15,16}$ reversibility after GERD therapy. ${ }^{17}$ However, a recent study did not find dilated ICS in patients with GERD and chronic laryngitis, posing a question whether dilated ICS occurs uniformly in patients with GERD. More recently, ICS was demonstrated to vary among subdivided layers of esophageal mucosa, suggesting the need for reconsideration of studying TEM by individual layer of esophageal muco$\mathrm{sa}^{18}$ although earlier studies did not perform TEM analysis of ICS in such manner. ${ }^{10,15}$

According to these considerations, the aims of this study were to characterize intercellular junction alterations and ICS of esophageal mucosa as measured by TEM and to investigate TJP of esophageal epithelium in patients with GERD.

\section{Materials and Methods}

\section{Subjects}

The study protocol was approved by Research Ethics Committee of Tzu Chi Medical Center. Each subject agreeing to participate provided informed consent. GERD patients with heartburn and/or acid regurgitation of at least 6 months' duration and history of response to proton pump inhibitor (PPI) were enrolled in the study. Asymptomatic controls were selected on the basis of absence of reflux symptoms and inflammation in the distal esophagus and undergoing EGD for other clinical reasons or healthy screening. Asymptomatic controls had no history of reflux disease or esophageal disease, and showed a grossly normal esophagus on endoscopy. Patients were excluded if they had the following conditions: (1) Barrett's esophagus, (2) esophageal strictures, (3) previous gastrointestinal surgery, and (4) concurrent use of PPI and/or other antisecretory agents. All subjects withheld acid suppressive therapy such as PPI and histamine type 2 receptor antagonists 4 weeks before EGD evaluation.

\section{Endoscopic Evaluation}

We evaluated the distal portion of the esophagus during standard EGD to determine the presence of mucosal injury. The extent of mucosal damage was assessed using the Los Angeles grading system. ${ }^{19} \mathrm{EE}$ was defined by the presence of endoscopically detectable mucosal breaks (erosions or ulcer), while the diagnosis of NERD was based on no endoscopically detectable mucosal lesions such as erosions or ulcers. Patients with EE were further subclassified into mild EE if their EGD showed grade A esophagitis, and moderate EE if EGD showed at least grade B. During the EGD, esophageal biopsies were obtained at $5 \mathrm{~cm}$ 
above the gastroesophageal junction for all subjects. Endoscopic biopsies were taken from endoscopic normal-looking mucosa.

\section{Immunohistochemical Analysis}

Five-micrometer paraffin-embedded sections were deparaffinized and rehydrated. Antigen retrieval was achieved by submerging the slides in citrate buffer $(\mathrm{pH}$ 6.0) and then overlaying them with a blocking reagent ( $1 \%$ bovine serum albumin) to prevent non-specific binding. After being washed with phosphate buffered saline (PBS), these sections were incubated with a rabbit anti-claudin-1 antibody (Zymed Laboratories, South San Francisco, CA, USA) or rabbit anti-claudin-2 antibody (Zymed) for 90 minutes at room temperature. After washing, the slides were stained with a biotinylated universal secondary antibody (Vectastain Elite ABC Kit; Vector, Burlingame, CA, USA), followed by further incubation with the avidin-biotin-peroxidase complex. Finally, these sections were immersed in 3-amino-9-ethylcarbazole (AEC Substrate kit; Vector) and counterstained with Mayer's hematoxylin. The degree of immunostaining was evaluated by pathologists and scored as follows: 0 , negative; $+1,<$ $10 \%$ of the cells with positive immunostaining; $+2,10-50 \%$ of the cells with positive immunostaining; +3 , greater than $50 \%$ of the cells with positive immunostaining.

\section{Transmission Electron Microscopy}

Biopsy specimens were immediately put in test tubes which contained fresh cold fixative. After three buffer rinses, the specimens were forefixed in $2.5 \%$ glutaraldehyde in $0.1 \mathrm{M}$ phosphate buffer ( $\mathrm{pH} 7.3$ ) for 4 hours, and then postfixed in $1 \%$ OsO4 for 2 hours. Samples were dehydrated in a graded ethanol-acetone series and embedded in Spurr resin. ${ }^{20}$ Thick sections $(1 \mu \mathrm{m})$ were stained with $1 \%$ tolutine blue in $1 \%$ borax at $60^{\circ} \mathrm{C}$, and thin sections $(90 \mathrm{~nm}$ ) were stained with uranyl acetate and lead citrate. LM and TEM examinations were accomplished with Leica Microscope and Hitachi-600 Transmission Electron Microscope.

\section{Intercellular Space Measurement and Data Analysis With Statistics}

All specimens were examined and were taken ten random digital micrographs (magnification of $\times 10,000$ ) from each group. From each photomicrography, 10 perpendicular lines were drawn, and diameter of ICS was utilized for measuring each TEM photomicrography with computerized image analysis program (Image Pro Plus, Ver.5.1; Media Cybernetics, Silver
Spring, MD, USA). Data were expressed as mean \pm SD. Groups' means were calculated using Minitab 15th ANOVA test and Tukey's multiple comparison assessment.

\section{Results}

\section{Experiment 1. Immunohistochemical Evalua- tion of Tight Junction Structure}

Esophageal biopsy specimens were obtained from 6 asymptomatic controls, and 12 GERD patients, of whom 6 had NERD, 3 had mild EE, and 3 had moderate EE. There were no statistical differences in the presence of claudin-1 and claudin-2 expressions among patients with moderate $\mathrm{EE}(2.5 \pm 1.2)$, mild $\mathrm{EE}(2.0 \pm 1.3), \mathrm{NERD}(2.3 \pm 1.3)$ and asymptomatic controls $(2.6 \pm 1.5)(P=\mathrm{NS})$. The immunohistochemical results revealed a wide and high intensity of membrane staining in all biopsies using an antibody against claudin- 1 and claudin-2 in GERD patients and asymptomatic controls. For example, the apical lo-

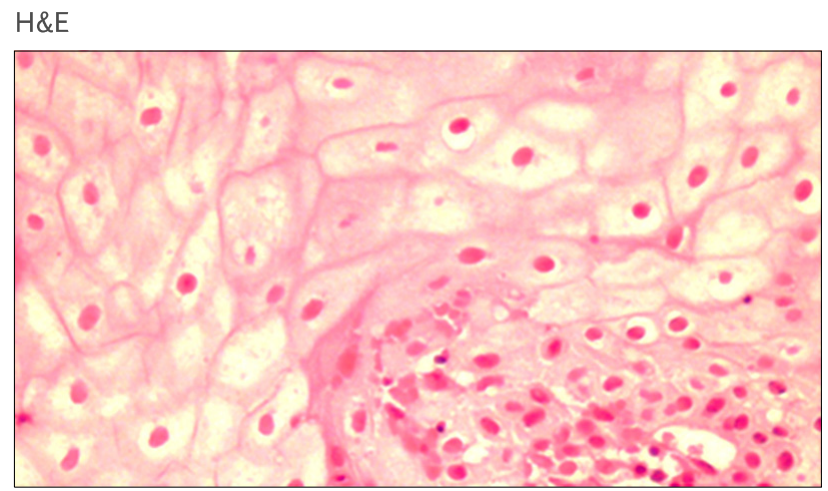

Claudin-1

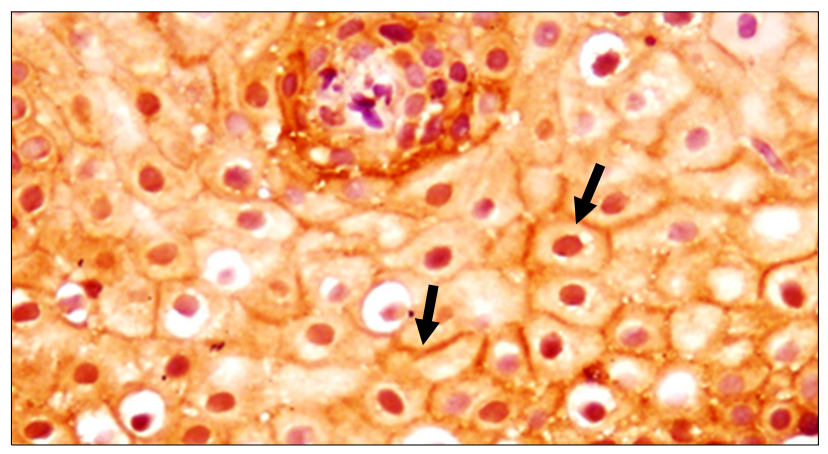

Figure 1. Claudin-1 expression in esophageal epithelium of patients with gastroesophageal reflux disease. The representative stain of claudin-1 showed a membrane-boundary pattern (indicated as arrows) (H\&E stain, $\times 200)$. 
calization of claudin-1 was clearly shown with membrane-boundary pattern (Fig. 1).

\section{Experiment 2. Transmission Electron Micro- scopy Evaluation of Epithelial Morphology and Intercellular Space}

In this experiment, we took esophageal biopsy specimens from 6 asymptomatic controls (4 male; mean age, 48.5; range, 21-60 years), 10 NERD patients ( 3 male; mean age, 45.9 ; range, 21-59 years), 6 mild EE patients (4 male; mean age, 52.3; range, 29-70 years) and 12 moderate EE patients (9 male; mean age, 51.4; range, 28-73 years). The displacement of upper stratified squamous and lower basal cuboidal epithelium did not show any relevant alteration in asymptomatic controls (Fig. 2A) and patients (Fig. 2B) in LM.

Measuring the ICS revealed a statistically significant difference in the mean diameter of ICS between upper squamous and lower basal cuboidal epithelium for each group of the subjects (all $P<0.001)$ (Table). The ICS of upper stratified squamous epithelium was significantly greater in patients with moderate EE when compared with the other 3 groups (all $P<0.001$ ) (Table).
The ICS of lower basal cuboidal epithelium did not differ among different patient groups and asymptomatic controls $(P=\mathrm{NS})$ (Table).

The ultrastructural characteristics of lower basal cuboidal epithelium did not show any relevant alteration between controls and GERD patients (Fig. 3C and 3D). In contrast, the desmosomal junctions and processes of upper stratified epithelium were different between asymptomatic controls and GERD patients. The ultrastructure of upper stratified squamous epithelium was normal with compact desmosomal junctions and intact processes in asymptomatic controls (Fig. 4A). The processes of desmosomal junctions at luminal side were impaired and blurred in some upper stratified squamous epithelium of patients with NERD (Fig. 4B). In addition, the processes of desmosomal junctions were significantly impaired and blurred in the majority of upper stratified squamous epithelium of patients with mild EE (Fig. 4C). In patients with moderate EE, most of desmosomal junctions demonstrated wider and increased ICS in upper stratified squamous epithelium (Fig. 4D).
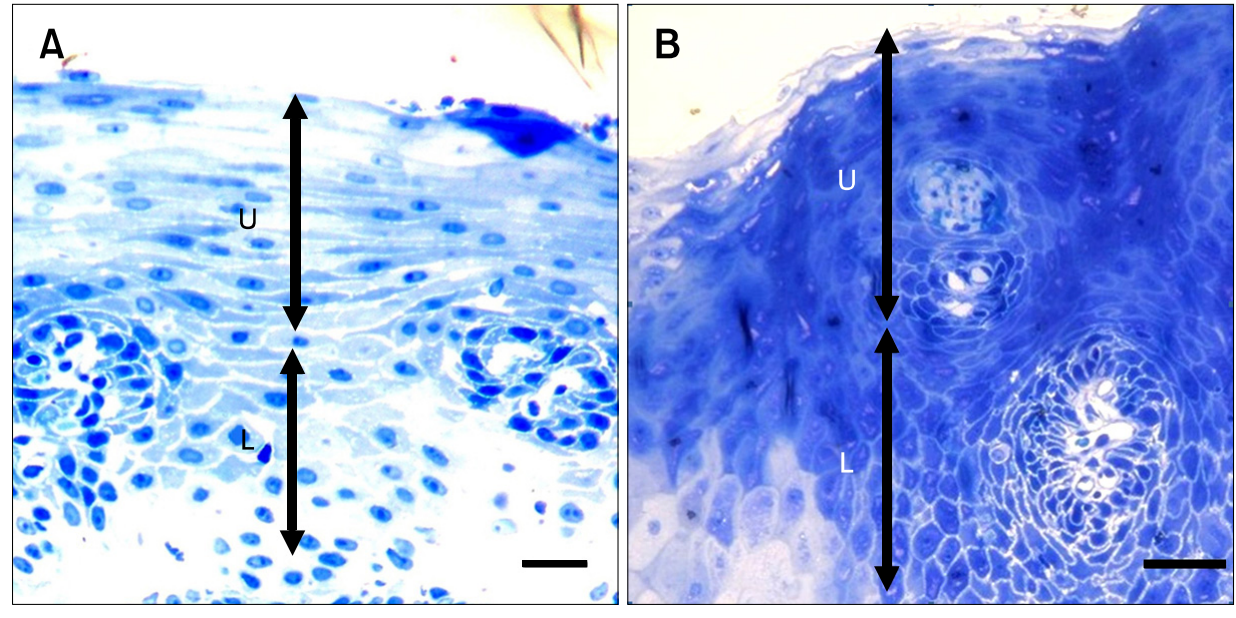

Figure 2. Tolutine blue stain of esophageal mucosa in asymptomatic controls (A) and patients (B) showing upper stratified squamous and lower basal cuboidal epithelium. Bar $=5 \mu \mathrm{m}(\times 200$ magnification). $U$, upper stratified squamous; L, lower basal cuboidal epithelium.

Table. Diameter of Intercellular Space of 4 Groups of Subjects in Upper Stratified Squamous and Lower Basal Cuboidal Epithelium

\begin{tabular}{lcccc}
\hline & $\begin{array}{c}\text { Asymptomatic } \\
\text { controls }(\mathrm{n}=6)\end{array}$ & $\begin{array}{c}\text { NERD } \\
(\mathrm{n}=10)\end{array}$ & $\begin{array}{c}\text { Mild EE } \\
(\mathrm{n}=6)\end{array}$ & $\begin{array}{c}\text { Moderate EE } \\
(\mathrm{n}=12)\end{array}$ \\
\hline Upper stratified squamous cells $(\mu \mathrm{m})$ & $0.514 \pm 0.109^{\mathrm{a}}$ & $0.519 \pm 0.132^{\mathrm{a}}$ & $0.591 \pm 0.121^{\mathrm{a}}$ & $1.078 \pm 0.231^{\mathrm{a}, \mathrm{b}}$ \\
Lower basal cuboidal cells $(\mu \mathrm{m})$ & $1.309 \pm 0.410$ & $1.309 \pm 0.410$ & $1.401 \pm 0.411$ & $1.649 \pm 0.408$ \\
\hline
\end{tabular}

NERD, non-erosive reflux disease; EE, erosive esophagitis.

${ }^{a} P<0.001$, upper stratified squamous cells vs. lower basal cuboidal cells for each group; ${ }^{\mathrm{b}} P<0.001$, moderate EE vs. any of the other groups for upper stratified squamous cells.

Data are shown as mean $\pm \mathrm{SD}$. 

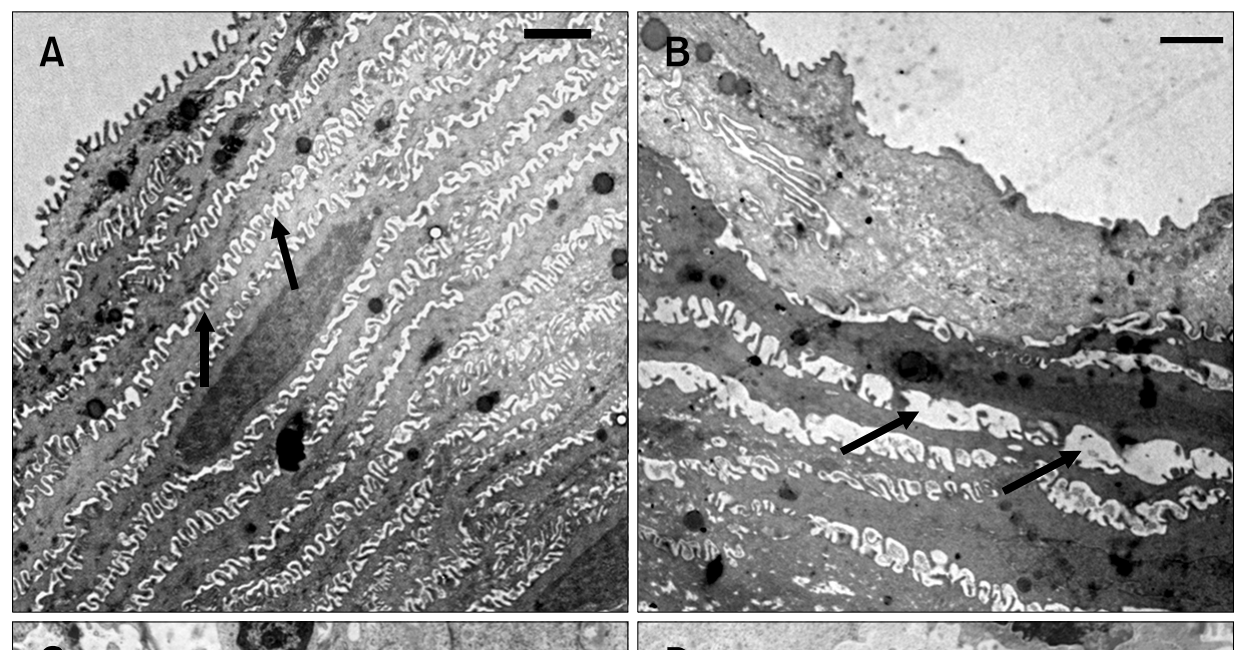

Figure 3. Electron micrographs showing the change of intercellular space (ICS) of the upper stratified squamous
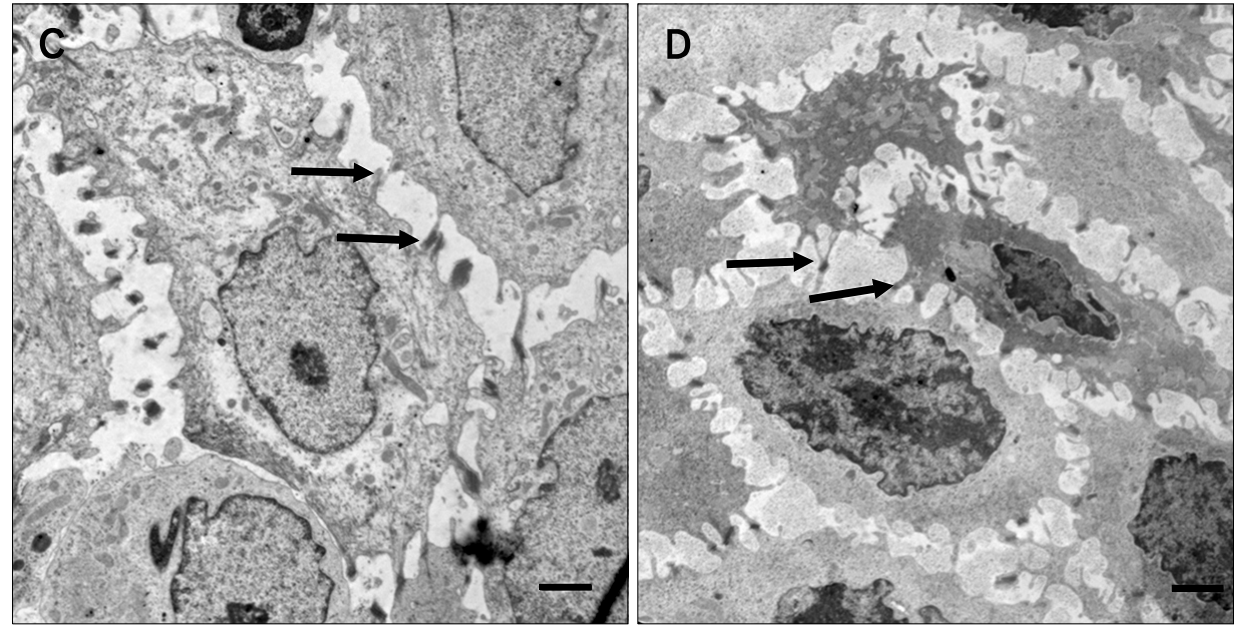
and lower basal cuboidal epithelium in asymptomatic controls and moderate erosive esophagitis (EE) patients. The ICS diameter of upper stratified squamous epithelium is shown in asymptomatic controls (A) and moderate EE patients (B). Clearly, the ICS diameter is larger in moderate $\mathrm{EE}$ patients. Bar $=1$ $\mu \mathrm{m}(\times 5,000$ magnification $)$. The diameter of ICS of lower basal cuboidal epithelium is similar between asymptomatic controls (C) and moderate EE patients (D). The desmosomal junctions and processes are intact (arrows) in both groups. Bar $=2.5 \mu \mathrm{m}(\times 10,000$ magnification).

\section{Discussion}

In this study, we observed that TJP such as claudin-1 and claudin-2 were fairly abundant not only in asymptomatic controls but also in patients with GERD. These morphological features suggested that the paracellular barrier function and cellular polarity did not change regardless of the different severity of GERD. We found that a significant ICS dilatation occurred only in upper stratified squamous epithelium of patients with moderate EE, but we did not demonstrate any increase in ICS of lower basal cuboidal epithelium among different groups of patients and asymptomatic controls. For all subjects, ICS appears to be greater in upper stratified squamous epithelium as compared with lower basal cuboidal epithelium. Furthermore, our study revealed that alterations in desmosomal junctions and their processes under TEM increased in parallel with the severity of GERD.

The normal esophagus is characterized by a stratified squ- amous non-keratinised epithelium with three main cell types, including flattened functional cells, polygonal prickle cells, and columnar basal cells as identified in order from luminal site. ${ }^{21} \mathrm{~A}$ recent work has demonstrated that ICS may differ among the three layers of esophageal epithelium in normal subjects, ${ }^{18}$ suggesting the notion that it is important to consider layer variation and subclassification for the proper identification and measurement of the ICS. Therefore, we studied ultrastructural morphology and ICS by subdividing esophageal mucosa into upper stratified squamous epithelium and lower basal cuboidal epithelium.

Desmosomes play an important role in maintaining intercellular adhesion, ${ }^{22}$ and their frequency may differ among different layers of esophageal mucosa. There are numerous desmosomes around the periphery of the prickle cell layer with short simple processes by which a close cell contact and formation of ICS are caused. ${ }^{22}$ In the inflamed human esophagus, an early study has demonstrated morphological alteration relevant to desmosomes, including irregular loss of their processes and even dis- 

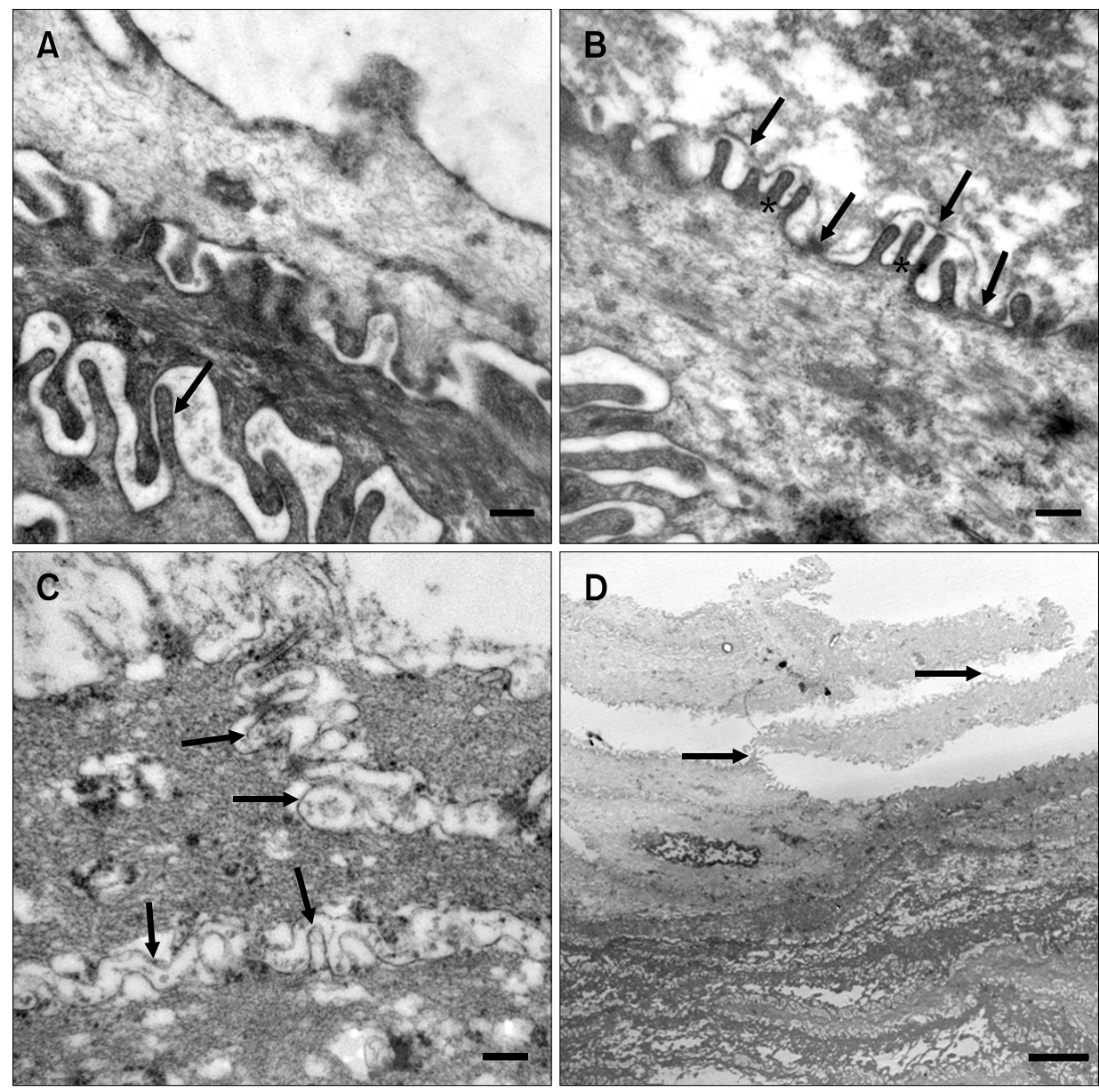

Figure 4. Electron micrograph showing the variations of desmosomal junctions and processes of upper stratified epithelium in asymptomatic controls and patients. (A) The desmosomal junctions and processes (arrows) were compact and intact in asymptomatic controls. Bar $=$ $0.5 \mu \mathrm{m}$ ( $\times 50,000$ magnification). (B) The processes of impaired cells were blurred (arrows), but the normal cells were intact (stars) in non-erosive reflux disease patients. Bar $=0.5 \mu \mathrm{m}(\times 50,000$ magnification). (C) The processes of impaired cells were blurred (arrows) in mild erosive esophagitis (EE) patients. Bar $=0.5 \mu \mathrm{m}(\times 50,000$ magnification $)$. (D) Most of desmosomal junctions of impaired cells were demonstrated wider (arrows) in moderate EE patients. Bar $=$ $5 \mu \mathrm{m}(\times 5,000$ magnification $)$. appearance of their structures, ${ }^{23}$ which were likely due to passage of inflammatory cell via ICS near superficial epithelium. ${ }^{23}$ In agreement with these findings, we also observed a progressive change regarding desmosomes which appear to exhibit gradually increased loss of either their structures or processes in the order from NERD patients to patients with severe esophageal inflammation. In patients with severe esophagitis, desmosomes and their processes almost disappeared with a significant increase in ICS, suggesting the loss in intercellular desmosomes might play a role in the enlargement of ICS as seen in our patients.

Tight junctions are positioned at the boundary of the apical and basolateral plasma membranes and play an important role in the paracellular barrier and cell polarity. ${ }^{24}$ It is suggested that tight junctions are crucial for the barrier function, ${ }^{24}$ and therefore the alterations in TJP may increase the permeability of the esophageal epithelium, thereby impairing the defense mechanism of the epithelium. We revealed that expressions of both claudin-1 and claudin- 2 proteins were abundant in asymptomatic controls as well as in patients with GERD. Our finding are in line with a previous study which showed claudin-1 in normal esophageal epithelium in patients with Barrett's esophagus. ${ }^{25} \mathrm{We}$ first observed that claudin-2 was actually detectable in both normal and inflamed esophageal mucosa, in contrast to an earlier study demonstrating its absence in human esophagus. ${ }^{25}$ On the other hand, in inflamed esophagus we observed the presence of claudin-1, which was also consistent with a previous work in that increased claudin-1 expression was found in inflamed esophagus of rat. ${ }^{26}$ Taken together with these findings, tight junction as part of intercellular junction complex appears to be normal and maintainable in patients with GERD regardless of clinical severity.

In contrast to previous findings of dilated ICS in erosive GERD $^{13,15,17}$ and NERD, ${ }^{16,17}$ we did not observe an equivalent increase of ICS in patients with GERD; however, only those with significant esophagitis were found to have dilated ICS. Our results are partially in line with a study on patients with GERD and chronic pharyngitis, which did not demonstrate increased ICS in patients with GERD, ${ }^{27}$ although the patients in their study comprised different population from NERD to severe EE. There are 
some probable explanations which can clarify the discrepancy between our findings and previous data. ${ }^{13,15-17,27}$ First, previous studies measuring ICS have omitted layer subclassification of esophageal epithelium and overlooked the fact that the mucosa layer can be further categorized into different layers, ${ }^{10,22}$ in which ICS might differ among different layers. ${ }^{18}$ Second, it is yet unclear whether acid reflux will produce a uniform damage to each subclassified layer of lower esophageal mucosa, and hence esophageal biopsy may not detect subtle changes in ICS. Similarly, ICS can also be different by a small distance near the endoscopic esophagitis. ${ }^{28,29}$ Third, differences in the measurement techniques and locations of biopsy site for ICS may also contribute to diverse results as compared with earlier reports. ${ }^{13,15-17}$ Lastly, we avoid observation bias by studying ultrastructure and ICS in a blind fashion in which TEM was performed without knowledge of each subject's diagnosis. It has been questioned about universal presence of ICS as depicted by Vaezi et al, ${ }^{27}$ who proposed that dilated ICS may not be specific to GERD alone. This is due to the fact that although dilated ICS is a sensitive marker of GERD, ${ }^{4}$ it can also be found in other clinical conditions such as psychological stress, ${ }^{10}$ food allergy ${ }^{30}$ and even after hypertonic luminal stimulation. ${ }^{31}$ In our study, we performed biopsy at fixed level, TEM evaluation and ICS measurement by separate mucosa layer, and blind ICS assessment and analysis. Thus, our findings may be a potential implication for further study that determines ICS more cautiously with particular emphasis on mucosal subclassification in the esophagus.

There are some limitations in this study. First, the subjects in our control group were not healthy volunteers, but patients with clinical diagnosis other than GERD. Therefore, possible asymptomatic reflux cannot be excluded. Nevertheless, with the absence of endoscopic esophagitis and typical reflux symptoms, the values for ICS may likely present those for healthy controls. Secondly, it is possible that the intercellular morphology may change along the esophagus, ${ }^{29}$ which may influence current data as we performed esophageal biopsies at $5 \mathrm{~cm}$ above the gastroesophageal junction. However, our data are similar to other studies using the same protocol. ${ }^{13,16,27,32}$ Since esophageal epithelial changes in response to acid reflux are patchy, specimens were taken at a constant distance $(5 \mathrm{~cm})$ proximal to the squamocolumnar junction, and areas of visible erosive lesions were avoided to minimize the sample error. The data regarding desmosomes were not quantitative by their presentation, and we only described their morphology alteration instead of applying quantitative comparisons with measurement tools. It is also important to evaluate the desmo- somes and their processes by using statistical analysis with standardized measurement tools. Finally, we only demonstrated the localization and expression patterns of claudin- 1 and claudin-2, although the tight junction consists of several other proteins including different subtypes of claudin, occludins, and junctional adhesion molecules. ${ }^{33}$ Future studies are needed to determine their role in tight junction in the esophageal mucosa.

In summary, TJP, claudin-1 and claudin-2, between esophageal epithelium did not change in patients with GERD independent from the presence of endoscopic esophagitis. The ultrastructural findings of desmosomal morphology alteration seem to be associated with increased severity of GERD. Our results do not show dilated ICS in GERD except for those with significant endoscopic esophagitis. This study supports a novel notion that dilated ICS may not be uniformly present inside esophageal mucosa and predominantly is located in upper squamous epithelium near the luminal site. However, the approach by examining each layer of esophageal epithelium for ultrastructure evaluation using TEM awaits further investigation regarding its validity and clinical relevance in patients with GERD.

\section{Acknowledgements}

We thank the Electron Microscopy Laboratory of Tzu Chi University for the TEM evaluation.

\section{References}

1. Dent J, El-Serag HB, Wallander MA, Johansson S. Epidemiology of gastro-oesophageal reflux disease: a systematic review. Gut 2005;54:710-717.

2. Frye JW, Vaezi MF. Extraesophageal GERD. Gastroenterol Clin North Am 2008;37:845-858, ix.

3. Johnsson F, Joelsson B, Gudmundsson K, Greiff L. Symptoms and endoscopic findings in the diagnosis of gastroesophageal reflux disease. Scand J Gastroenterol 1987;22:714-718.

4. Orlando LA, Orlando RC. Dilated intercellular spaces as a marker of GERD. Curr Gastroenterol Rep 2009;11:190-194.

5. Garrod D, Chidgey M. Desmosome structure, composition and function. Biochim Biophys Acta 2008;1778:572-587.

6. Niessen CM. Tight junctions/adherens junctions: basic structure and function. J Invest Dermatol 2007;127:2525-2532.

7. Morita K, Furuse M, Fujimoto K, Tsukita S. Claudin multigene family encoding four-transmembrane domain protein components of tight junction strands. Proc Natl Acad Sci USA 1999;96:511-516.

8. Turksen K, Troy TC. Barriers built on claudins. J Cell Sci 2004; 117(Pt 12):2435-2447.

9. Van Itallie CM, Fanning AS, Anderson JM. Reversal of charge selectivity in cation or anion-selective epithelial lines by expression of 
different claudins. Am J Physiol Renal Physiol 2003;285:F1078F1084.

10. Farré R, De Vos R, Geboes K, et al. Critical role of stress in increased oesophageal mucosa permeability and dilated intercellular spaces. Gut 2007;56:1191-1197.

11. Carney CN, Orlando RC, Powell DW, Dotson MM. Morphologic alterations in early acid-induced epithelial injury of the rabbit esophagus. Lab Invest 1981;45:198-208.

12. Tobey NA, Orlando RC. Mechanisms of acid injury to rabbit esophageal epithelium. Role of basolateral cell membrane acidification. Gastroenterology 1991;101:1220-1228.

13. Tobey NA, Carson JL, Alkiek RA, Orlando RC. Dilated intercellular spaces: a morphological feature of acid reflux - damaged human esophageal epithelium. Gastroenterology 1996;111:1200-1205.

14. Calabrese C, Fabbri A, Bortolotti M, et al. Dilated intercellular spaces as a marker of oesophageal damage: comparative results in gastro-oesophageal reflux disease with or without bile reflux. Aliment Pharmacol Ther 2003;18:525-532.

15. Caviglia R, Ribolsi M, Gentile M, et al. Dilated intercellular spaces and acid reflux at the distal and proximal oesophagus in patients with non-erosive gastro-oesophageal reflux disease. Aliment Pharmacol Ther 2007;25:629-636.

16. Caviglia R, Ribolsi M, Maggiano N, et al. Dilated intercellular spaces of esophageal epithelium in nonerosive reflux disease patients with physiological esophageal acid exposure. Am J Gastroenterol 2005;100:543-548.

17. Calabrese C, Bortolotti M, Fabbri A, et al. Reversibility of GERD ultrastructural alterations and relief of symptoms after omeprazole treatment. Am J Gastroenterol 2005;100:537-542.

18. Park S, Chun HJ, Jang JS, et al. Is intercellular space different among layers in normal esophageal mucosa? An electron microscopic study. Dig Dis Sci 2011;56:3492-3497.

19. Lundell LR, Dent J, Bennett JR, et al. Endoscopic assessment of oesophagitis: clinical and functional correlates and further validation of the Los Angeles classification. Gut 1999;45:172-180.

20. Spurr AR. A low-viscosity epoxy resin embedding medium for electron microscopy. J Ultrastruct Res 1969;26:31-43.

21. Hopwood D, Logan KR, Bouchier IA. The electron microscopy of normal human oesophageal epithelium. Virchows Arch B Cell Pathol
1978;26:345-358.

22. Logan KR, Hopwood D, Milne G. Cellular junctions in human oesophageal epithelium. J Pathol 1978;126:157-163.

23. Hopwood D, Milne G, Logan KR. Electron microscopic changes in human oesophageal epithelium in oesophagitis. J Pathol 1979;129: 161-167.

24. Tsukita S, Furuse M. Claudin-based barrier in simple and stratified cellular sheets. Curr Opin Cell Biol 2002;14:531-536.

25. Rendon-Huerta E, Valenzano MC, Mullin JM, et al. Comparison of three integral tight junction barrier proteins in Barrett's epithelium versus normal esophageal epithelium. Am J Gastroenterol 2003;98: 1901-1903.

26. Asaoka D, Miwa H, Hirai S, et al. Altered localization and expression of tight-junction proteins in a rat model with chronic acid reflux esophagitis. J Gastroenterol 2005;40:781-790.

27. Vaezi MF, Slaughter JC, Smith BS, et al. Dilated intercellular space in chronic laryngitis and gastro-oesophageal reflux disease: at baseline and post-lansoprazole therapy. Aliment Pharmacol Ther 2010;32: 916-924.

28. Edebo A, Vieth M, Tam W, et al. Circumferential and axial distribution of esophageal mucosal damage in reflux disease. Dis Esophagus 2007;20:232-238.

29. Vieth M, Fiocca R, Haringsma J, et al. Radial distribution of dilated intercellular spaces of the esophageal squamous epithelium in patients with reflux disease exhibiting discrete endoscopic lesions. Dig Dis 2004;22:208-212.

30. Ravelli AM, Villanacci V, Ruzzenenti N, et al. Dilated intercellular spaces: a major morphological feature of esophagitis. J Pediatr Gastroenterol Nutr 2006;42:510-515.

31. Long JD, Marten E, Tobey NA, Orlando RC. Effects of luminal hypertonicity on rabbit esophageal epithelium. Am J Physiol 1997;273(3 Pt 1):G647-G654.

32. Vela MF, Craft BM, Sharma N, Freeman J, Hazen-Martin D. Refractory heartburn: comparison of intercellular space diameter in documented GERD vs. functional heartburn. Am J Gastroenterol 2010;106:844-850.

33. Kimura Y, Shiozaki H, Hirao M, et al. Expression of occludin, tight-junction-associated protein, in human digestive tract. Am J Pathol 1997;151:45-54. 